\title{
Space-time inhomogeneous quantum random walk in terms of quantum Bernoulli noise
}

\author{
Meng Li, Caishi Wang, Rui Fu
}

\begin{abstract}
The evolution equation of the Space-time inhomogeneous quantum random walk in terms of quantum Bernoulli noise not only depends on the change of time, but also on the spatial position of the walker. The model is defined on the space of square summable functions. The canonical isomorphism relationship also can deduce the representation of the model in the tensor space. Mathematical induction gives the probability distribution of the quantum random walk in the general initial state and find that it has the same limit distribution as the classical random walk.
\end{abstract}

Keywords-quantum Bernoulli noise; Space-time inhomogeneous quantum random walk; probability distribution

\section{INTRODUCTION}

Classical random walks [1] has been widely used in the fields of computer science, economics, physics and biology. With the upsurge of research on quantum information theory, people began to extend the classical random walks theory to quantum systems, forming a new walk model-quantum random walks[2]. As quantum analogs of classical random walks, quantum walks have found wide application in quantum information, information, quantum computing and many other fields[3]-[5]. In recent years, quantum random walks have been intensively studied and many deep results have been obtained received more and more attention and research, and many profound results have been obtained[3]-[8].

Quantum Bernoulli noises refer to the family of annihilation and creation operators acting on the space of square-integrable Bernoulli functionals, which satisfy a canonical anti-commutation relation (CAR) in equal time. the concept is proposed in [9], By using quantum Bernoulli noise, Wang an Ye introduced a discrete-time quantum walk model on the one dimensional integer lattice $\mathbb{Z}(\mathrm{W}-\mathrm{Y}$ quantum random walk), it has the same limit probability distribution as the classical random walk [10]. For a general positive integer $d \geqslant 2$, by using quantum Bernoulli noise, [11] introduces a model of discrete time quantum walk on the d-dimensional integer lattice $\mathbb{Z}^{\mathrm{d}}$, which we call the d-dimensional QBN walk, Moreover proved that,the d-dimensional QBN walk has a limit probability distribution of d-dimensional standard Gauss type. [12] uses quantum Bernoulli noise to introduces open quantum random walks(OQRW, for short), and studies the related proper- ties of OQRW from the perspective of random walk.

$\mathrm{W}-\mathrm{Y}$ quantum random walk is a quantum random walk inhomogeneous in time. The evolution equation depends on the change of time, the cion operator are $\mathrm{L}_{\mathrm{n}}=\frac{1}{2}\left(\partial_{\mathrm{n}}^{*}+\partial_{\mathrm{n}}-\mathrm{I}\right)$ and $\mathrm{R}_{\mathrm{n}}=\frac{1}{2}\left(\partial_{\mathrm{n}}^{*}+\partial_{\mathrm{n}}+\mathrm{I}\right)$. Wada considereds a quantum random walk non-homogeneous in space[13]. The evolution equation depends on the spatial position of the walk, the coin operator are

$$
\begin{aligned}
& C(x)=e^{-i \xi(x)}\left[\begin{array}{ll}
1 & 0 \\
0 & 0
\end{array}\right] C_{0} \\
& \text { 和 } D(x)=e^{i \xi(x)}\left[\begin{array}{ll}
0 & 0 \\
0 & 1
\end{array}\right] C_{0},
\end{aligned}
$$

where $\xi: \mathbb{Z} \rightarrow \mathbb{R}, C_{0} \in U(2)$ and $C(x)+D(x)$ is the unitary operator. Inspired by [10] and [13], we introduction a new quantum walk in which both time and space are inhomogeneous, its evolution equation is not only related to the change of time, but also related to the spatial position of the walk. Use the operator $L_{n}(x)=$ $\mathrm{e}^{-\mathrm{i} \xi(\mathrm{x})} \frac{1}{2}\left(\partial_{\mathrm{n}}^{*}+\partial_{\mathrm{n}}-\mathrm{I}\right)$ and $\mathrm{R}_{\mathrm{n}}(\mathrm{x})=\mathrm{e}^{\mathrm{i} \xi(\mathrm{x})} \frac{1}{2}\left(\partial_{\mathrm{n}}^{*}+\partial_{\mathrm{n}}+\right.$ I) acts as a coin operator to construct a space-time inhomogeneous quantum random walk, and study its dynamic properties.

\section{PRELIMINARIES}

In this section, we briefly recall some necessary notions and facts about quantum Bernoulli noises. Throughout this paper, $\mathbb{Z}$ always denotes the set of all integers, which $\mathbb{N}$ means the set of all nonnegative integers. We denotes by $\Gamma$ the finite power of $\mathbb{N}$, namely

$$
\Gamma=\{\sigma \mid \sigma \in \mathbb{N} \text { 且 } \# \sigma<\infty\},
$$

Where $\# \sigma$ means the cardinality of $\sigma$. Unless otherwise stated, letters like $\mathrm{j}, \mathrm{k}$ and $\mathrm{n}$ stand for nonnegative integers, namely elements of $\mathbb{N}$.

$(\Omega, \mathcal{F}, \mathrm{P})$ is a probability measurement space, called Bernoulli space, which satisfies:

(1) $\Omega=\{-1,1\}^{\mathbb{N}}$ means the set of all mappings $\omega: \mathbb{N} \mapsto\{-1,1\}$.

(2) (2) $\left(\zeta_{n}\right)_{n \geq 0}$ means the sequence of canonical projections on $\Omega$ given by

$$
\zeta_{n}(\omega)=\omega(n), \omega \in \Omega .
$$

$\mathcal{F}$ be the $\sigma$-field on $\Omega$ generated by the sequence $\left(\zeta_{n}\right)_{n \geq 0}$. 
(3) $\left(p_{n}\right)_{n \geq 0}$ be a given sequence of positive number with the property that $0<p_{n}<1$ for all $n \geq 0$. Then there exist a unique probability measure $\mathrm{P}$ on $\mathcal{F}$ such that[14]

$$
\begin{aligned}
& P \circ\left(\zeta_{n_{1}}, \zeta_{n_{2}}, \cdots \zeta_{n_{k}}\right)^{-1}\left\{\left(\varepsilon_{1}, \varepsilon_{2}, \cdots \varepsilon_{k}\right)\right\} \\
& =\prod_{j=1}^{k} p_{n_{j}^{2}}^{\frac{1+\varepsilon_{j}}{2}} q_{n_{j}^{2}},
\end{aligned}
$$

for $\quad n_{j} \in \mathbb{N}, \varepsilon_{j} \in\{-1,1\},(1 \leq j \leq k) \quad$ with $\quad n_{i} \neq n_{j}$ when $\mathrm{i} \neq \mathrm{j}$ and $\mathrm{k} \in \mathbb{N}$ with $\mathrm{k} \geq 1$.

Let $\mathbb{Z}=\left(\mathbb{Z}_{n}\right)_{n \geq 0}$ be the sequence of Bernoulli functionals generated by sequence $\left(\zeta_{\mathrm{n}}\right)_{\mathrm{n} \geq 0}$, namely

$$
\mathbb{Z}_{\mathrm{n}}=\frac{\zeta_{\mathrm{n}}+\mathrm{q}_{\mathrm{n}}-\mathrm{p}_{\mathrm{n}}}{2 \sqrt{\mathrm{p}_{\mathrm{n}} \mathrm{q}_{\mathrm{n}}}}, \mathrm{n} \geq 0,
$$

where $\mathrm{q}_{\mathrm{n}}=1-\mathrm{p}_{\mathrm{n}}$. Clearly $\mathbb{Z}=\left(\mathbb{Z}_{\mathrm{n}}\right)_{\mathrm{n} \geq 0}$ is an independent sequence of random variables on the probability measure space $(\Omega, \mathcal{F}, \mathrm{P})$. and for each $\mathrm{n} \geq 0, \mathbb{Z}_{\mathrm{n}}$ has a distribution

$\mathrm{P}\left\{\mathbb{Z}_{\mathrm{n}}=\theta_{\mathrm{n}}\right\}=\mathrm{p}_{\mathrm{n}}, \mathrm{P}\left\{\mathbb{Z}_{\mathrm{n}}=-1 / \theta_{\mathrm{n}}\right\}=\mathrm{q}_{\mathrm{n}}, \mathrm{n} \geq 0$, with $\theta_{\mathrm{n}}=\sqrt{\frac{q_{\mathrm{n}}}{\mathrm{p}_{\mathrm{n}}}}$.

Let $\mathcal{H}$ be the space of square integrable complex-valued Bernoulli functionals, namely

$$
\mathcal{H}=\mathrm{L}^{2}(\Omega, \mathcal{F}, \mathrm{P}) .
$$

We denote by $\langle\cdot\rangle$,$\rangle the usual inner product of the$ space $\mathcal{H}$, and by $\|\cdot\|$ the corresponding norm. We further assume that $\mathbb{Z}$ has the chaotic representation property [14], which means $\left\{\mathbb{Z}_{\sigma} \mid \sigma \in \Gamma\right\}$ forms an orthonormal basis of $\mathcal{H}$, where $\mathbb{Z}_{\emptyset}=1$, and

$$
\mathbb{Z}_{\sigma}=\prod_{\mathrm{i} \in \sigma} \mathbb{Z}_{\mathrm{i}}, \sigma \in \Gamma, \sigma \neq \emptyset .
$$

Clearly $\mathcal{H}$ is infinite-dimensional as a complex Hilbert space.

Recall that $\mathcal{H}$ has an orthonormal basis $\left\{\mathbb{Z}_{\sigma} \mid \sigma \in \Gamma\right\}$. Thus, for each $\mathrm{n} \geq 0$, we can naturally introduce a subspace of $\mathcal{H}$ :

$$
\mathcal{H}_{\mathrm{n}}=\operatorname{span}\left\{\mathbb{Z}_{\sigma} \mid \mathrm{m} \leq \mathrm{n}, \sigma \in \Gamma\right\},
$$

namely the one spanned by $\left\{\mathbb{Z}_{\sigma} \mid \max \leq \mathrm{n}, \sigma \in \Gamma\right\}$. We additionally write $\mathcal{H}_{-1}=\operatorname{span}\left\{\mathbb{Z}_{\emptyset}\right\}$, which in actually the same as $\mathbb{C}$. It can be easilv checked that the dimension of $\mathcal{H}_{\mathrm{n}}$ is just $2^{\mathrm{n}+1}$.

Lemma 2.1.1 [9] For each $\mathrm{k} \geq 0$, there exists a bounded operator $\partial_{\mathrm{k}}$ and its adjoint operator $\partial_{\mathrm{k}}^{*}$ satisfies $\partial_{\mathrm{k}} \mathbb{Z}_{\sigma}$

$=1_{\sigma}(\mathrm{k}) \mathbb{Z}_{\sigma \backslash \mathrm{k}}, \partial_{\mathrm{k}}^{*} \mathbb{Z}_{\sigma}=\left[1-1_{\sigma}(\mathrm{k})\right] \mathbb{Z}_{\sigma \cup \mathrm{k}}, \quad \sigma \in \Gamma$, where $\sigma \backslash \mathrm{k}=\sigma \backslash\{\mathrm{k}\}, \sigma \cup \mathrm{k}=\sigma \cup\{\mathrm{k}\}$, and $1_{\sigma}(\mathrm{k})$ is the indicator of $\sigma$ as a subset of $\mathbb{N}$.

Definition 2.1.2 [10] The operator $\partial_{\mathrm{k}}^{*}$ and its adjoint $\partial_{\mathrm{k}}^{*}$ are usually known as the annihilation and creation operators acting on Bernoulli functionals. The family $\left\{\partial_{\mathrm{k}}, \partial_{\mathrm{k}}^{*}\right\}_{\mathrm{k} \geq 0}$ of creation and annihilation operators are called quantum Bernoulli noises.

Lemma 2.1.3 [10] Let $k, l \in \mathbb{N}$. Then, it holds true that $\partial_{\mathrm{k}} \partial_{\mathrm{l}}=\partial_{\mathrm{l}} \partial_{\mathrm{k}}, \partial_{\mathrm{k}}^{*} \partial_{\mathrm{l}}^{*}=\partial_{\mathrm{l}}^{*} \partial_{\mathrm{k}}^{*}, \partial_{\mathrm{k}}^{*} \partial_{1}=\partial_{1} \partial_{\mathrm{k}}^{*}(\mathrm{k} \neq \mathrm{l})$, and

$$
\partial_{\mathrm{k}} \partial_{\mathrm{k}}=\partial_{\mathrm{k}}^{*} \partial_{\mathrm{k}}^{*}=0, \partial_{\mathrm{k}} \partial_{\mathrm{k}}^{*}+\partial_{\mathrm{k}}^{*} \partial_{\mathrm{k}}=\mathrm{I},
$$

where $\mathrm{I}$ is the identity operator on $\mathcal{H}$.

Lemma 2.1.3 shows that quantum Bernoulli noises satisfy the canonical anti-commutation relations(CAR) in equal time.

\section{MAIN RESULTS}

In this section, we present the definition of our walk on the space of square summable functions by quantum Bernoulli noises and a real-valued function defined on integer lattice $\mathbb{Z}$.

\subsection{Definition of the model}

Let $l^{2}(\mathbb{Z}, \mathcal{H})$ be the space of square summable functions on $\mathbb{Z}$ and valued in $\mathcal{H}$. namely

$$
\mathrm{l}^{2}(\mathbb{Z}, \mathcal{H})=\left\{\Phi: \mathbb{Z} \rightarrow \mathcal{H} \mid \sum_{\mathrm{x}=-\infty}^{\infty}\|\Phi(\mathrm{x})\|^{2}<\infty\right\} .
$$

Then $l^{2}(\mathbb{Z}, \mathcal{H})$ remains a complex Hilbert space, whose inner product $\langle\cdot, \cdot\rangle_{1^{2}(\mathbb{Z}, \mathcal{H})}$ is given by

$$
\langle\Phi, \Psi\rangle_{1^{2}(\mathbb{Z}, \mathcal{H})}=\sum_{\mathrm{x}=-\infty}^{\infty}\langle\Phi(\mathrm{x}), \Psi(\mathrm{x})\rangle,
$$

where $\Phi, \Psi \in \mathrm{l}^{2}(\mathbb{Z}, \mathcal{H})$, and $\langle\cdot \cdot\rangle_{1^{2}(\mathbb{Z}, \mathcal{H})}$ denotes the inner product of $\mathcal{H}$, and denote by $\|\cdot\|_{1^{2}(\mathbb{Z}, \mathcal{H})}$ the corresponding norm.

Definition 3.1.1 For a nonnegative integer $n \geq 0$ and each $x \in \mathbb{Z}$, one can introduce two operators $R_{n}(x)$ and $L_{n}(x)$ :

$$
\begin{aligned}
& \mathrm{L}_{\mathrm{n}}(\mathrm{x})=\mathrm{e}^{-\mathrm{i} \xi(\mathrm{x})} \frac{1}{2}\left(\partial_{\mathrm{n}}^{*}+\partial_{\mathrm{n}}-\mathrm{I}\right) \\
& \mathrm{R}_{\mathrm{n}}(\mathrm{x})=\mathrm{e}^{\mathrm{i} \xi(\mathrm{x})} \frac{1}{2}\left(\partial_{\mathrm{n}}^{*}+\partial_{\mathrm{n}}+\mathrm{I}\right)
\end{aligned}
$$

where $\mathrm{I}$ is the identity operator on $\mathcal{H}, \xi(\mathrm{x})$ denotes a real-valued function defined on integer lattice $\mathbb{Z}$.

Remark 1: $\mathrm{R}_{\mathrm{n}}(\mathrm{x})$ and $\mathrm{L}_{\mathrm{n}}(\mathrm{x})$ are non-selfadjoint operators on space $\mathcal{H}$.

Definition 3.1.2 Set $\mathrm{L}_{\varnothing}=\mathrm{R}_{\varnothing}=\mathrm{I}$ and

$$
\begin{gathered}
\mathrm{L}_{\sigma}(\mathrm{x})=\prod_{\mathrm{k} \in \sigma} \mathrm{L}_{\mathrm{k}}(\mathrm{x}), \mathrm{R}_{\sigma}(\mathrm{x})=\prod_{\mathrm{k} \in \sigma} \mathrm{R}_{\mathrm{k}}(\mathrm{x}), \sigma \neq \varnothing .
\end{gathered}
$$

Theorem 3.1.3 Set ${ }^{(x)}$ denotes a real-valued function 
defined on integer lattice $\mathbb{Z}$, . for each $n \geq 0, R_{n}(x)+L_{n}(x)$ is an unitary operator on $\mathcal{H}$ and moreover

$$
\begin{gathered}
\mathrm{R}_{\mathrm{n}}^{*}(\mathrm{x}) \mathrm{L}_{\mathrm{n}}(\mathrm{x})=\mathrm{R}_{\mathrm{n}}(\mathrm{x}) \mathrm{L}_{\mathrm{n}}^{*}(\mathrm{x})=0, \\
\mathrm{x} \in \mathbb{Z}, \mathrm{n} \geq 0 .
\end{gathered}
$$

Proof. For each $n \geq 0$, let $T_{n}(x)=R_{n}(x)+$ $\mathrm{L}_{\mathrm{n}}(\mathrm{x})$, then have $\mathrm{T}_{\mathrm{n}}^{*}(\mathrm{x})=\mathrm{R}_{\mathrm{n}}^{*}(\mathrm{x})+\mathrm{L}_{\mathrm{n}}^{*}(\mathrm{x})$, and moreover

$$
\begin{aligned}
& T_{n}(x) T_{n}^{*}(x) \\
= & {\left[R_{n}(x)+L_{n}(x)\right]\left[R_{n}^{*}(x)+L_{n}^{*}(x)\right] } \\
= & \frac{1}{4}\left[2 I+2\left(\partial_{n}^{*}+\partial_{n}\right)\right]+\frac{1}{4}\left[2 I-2\left(\partial_{n}^{*}+\partial_{n}\right)\right] \\
= & I,
\end{aligned}
$$

similarly, we can verify $\mathrm{T}_{\mathrm{n}}^{*}(\mathrm{x}) \mathrm{T}_{\mathrm{n}}(\mathrm{x})=\mathrm{I}$. namely $\mathrm{R}_{\mathrm{n}}(\mathrm{x})+\mathrm{L}_{\mathrm{n}}(\mathrm{x})$ is a unitary operator. Moreover, for each $\mathrm{n} \geq 0$, we have

$$
\begin{aligned}
& \mathrm{R}_{\mathrm{n}}^{*}(\mathrm{x}) \mathrm{L}_{\mathrm{n}}(\mathrm{x}) \\
& =\left[\mathrm{e}^{\mathrm{i} \xi(\mathrm{x})} \frac{1}{2}\left(\partial_{\mathrm{n}}^{*}+\partial_{\mathrm{n}}+\mathrm{I}\right)\right]\left[\mathrm{e}^{-\mathrm{i} \xi(\mathrm{x})} \frac{1}{2}\left(\partial_{\mathrm{n}}^{*}+\partial_{\mathrm{n}}-\mathrm{I}\right)\right] \\
& =0=\mathrm{R}_{\mathrm{n}}(\mathrm{x}) \mathrm{L}_{\mathrm{n}}^{*}(\mathrm{x}) .
\end{aligned}
$$

Theorem 3.1.4 Let $n \geq 0, \Phi \in \mathrm{l}^{2}(\mathbb{Z}, \mathcal{H})$. If a function $\Psi: \mathbb{Z} \rightarrow \mathcal{H}$, satisfies condition

$\Psi(\mathrm{x})=\mathrm{R}_{\mathrm{n}}(\mathrm{x}-1) \Phi(\mathrm{x}-1)+\mathrm{L}_{\mathrm{n}}(\mathrm{x}+1) \Phi(\mathrm{x}+1)$,

where $\mathrm{x} \in \mathbb{Z}$, then $\Psi \in \mathrm{l}^{2}(\mathbb{Z}, \mathcal{H})$ and $\|\Psi\|_{1^{2}(\mathbb{Z}, \mathcal{H})}=\|$ $\Phi \|_{1^{2}(\mathbb{Z}, \mathcal{H})}$.

Proof. It follows from formula $\mathrm{R}_{\mathrm{n}}^{*}(\mathrm{x}) \mathrm{L}_{\mathrm{n}}(\mathrm{x})=$ $\mathrm{R}_{\mathrm{n}}(\mathrm{x}) \mathrm{L}_{\mathrm{n}}^{*}(\mathrm{x})=0$ that

$$
\begin{aligned}
& \sum_{\mathrm{x}=-\infty}^{\infty}\|\Psi(\mathrm{x})\|^{2} \\
= & \sum_{\mathrm{x}=-\infty}^{\infty}\left\langle\Phi(\mathrm{x}-1), \mathrm{R}_{\mathrm{n}}^{*}(\mathrm{x}-1) \mathrm{R}_{\mathrm{n}}(\mathrm{x}-1) \Phi(\mathrm{x}-1)\right\rangle+ \\
& \sum_{\mathrm{x}=-\infty}^{\infty}\left\langle\Phi(\mathrm{x}-1), \mathrm{R}_{\mathrm{n}}^{*}(\mathrm{x}-1) \mathrm{L}_{\mathrm{n}}(\mathrm{x}+1) \Phi(\mathrm{x}+1)\right\rangle+ \\
& \sum_{\mathrm{x}=-\infty}^{\infty}\left\langle\Phi(\mathrm{x}+1), \mathrm{L}_{\mathrm{n}}^{*}(\mathrm{x}+1) \mathrm{R}_{\mathrm{n}}(\mathrm{x}-1) \Phi(\mathrm{x}-1)\right\rangle+ \\
& \sum_{\mathrm{x}=-\infty}^{\infty}\left\langle\Phi(\mathrm{x}+1), \mathrm{L}_{\mathrm{n}}^{*}(\mathrm{x}+1) \mathrm{L}_{\mathrm{n}}(\mathrm{x}+1) \Phi(\mathrm{x}+1)\right\rangle \\
= & \sum_{\mathrm{x}=-\infty}^{\infty}\left\langle\Phi(\mathrm{x}), \mathrm{R}_{\mathrm{n}}^{*}(\mathrm{x}) \mathrm{R}_{\mathrm{n}}(\mathrm{x}) \Phi(\mathrm{x})\right\rangle+ \\
& \sum_{\mathrm{x}=-\infty}^{\infty}\left\langle\Phi(\mathrm{x}), \mathrm{L}_{\mathrm{n}}^{*}(\mathrm{x}) \mathrm{L}_{\mathrm{n}}(\mathrm{x}) \Phi(\mathrm{x})\right\rangle \\
= & \sum_{\mathrm{x}=-\infty}^{\infty}\langle\Phi(\mathrm{x}), \Phi(\mathrm{x})\rangle \\
= & \sum_{\mathrm{x}=-\infty}^{\infty}\|\Phi(\mathrm{x})\|,
\end{aligned}
$$

which together with the assumption $\Psi \in \mathrm{l}^{2}(\mathbb{Z}, \mathcal{H})$ means that $\|\Psi\|_{1^{2}(\mathbb{Z}, \mathcal{H})}=\|\Phi\|_{1^{2}(\mathbb{Z}, \mathcal{H})}$.

Definition3.1.5 The state space of Spatiotemporal non-homogeneous quantum random walk is $\mathrm{l}^{2}(\mathbb{Z}, \mathcal{H})$, the time evolution of the walk is governed by equation

$$
\begin{aligned}
& \Phi_{(\mathrm{n}+1)}(\mathrm{x}) \\
& =\mathrm{R}_{\mathrm{n}}(\mathrm{x}-1) \Phi_{\mathrm{n}}(\mathrm{x}-1)+\mathrm{L}_{\mathrm{n}}(\mathrm{x}+1) \Phi_{\mathrm{n}}(\mathrm{x}+1),
\end{aligned}
$$

where $\mathrm{x} \in \mathbb{Z}, \mathrm{n} \geq 0$, and $\Phi_{\mathrm{n}}$ means the state of the walk at the time $\mathrm{n} \geq 0$, and in particular $\Phi_{0}$ is the initial state of the walk.

Theorem 3.1.6 Let $\mathrm{n} \geq 0$, there exists a unitary operator $\mathrm{V}_{\mathrm{n}}: \mathrm{l}^{2}(\mathbb{Z}, \mathcal{H}) \rightarrow \mathrm{l}^{2}(\mathbb{Z}, \mathcal{H})$, such that

$$
\left[\mathrm{V}_{\mathrm{n}} \Phi\right](\mathrm{x})=\mathrm{R}_{\mathrm{n}}(\mathrm{x}-1) \Phi(\mathrm{x}-1)+\mathrm{L}_{\mathrm{n}}(\mathrm{x}+1) \Phi(\mathrm{x}+1),
$$

and

$\left[\mathrm{V}_{\mathrm{n}}^{*} \Phi\right](\mathrm{x})=\mathrm{R}_{\mathrm{n}}^{*}(\mathrm{x}) \Phi(\mathrm{x}+1)+\mathrm{L}_{\mathrm{n}}^{*}(\mathrm{x}) \Phi(\mathrm{x}-1)$, (3.7) where $\mathrm{x} \in \mathbb{Z}, \Phi \in \mathrm{l}^{2}(\mathbb{Z}, \mathcal{H})$, and $\mathrm{V}_{\mathrm{n}}^{*}$ denotes the adjoint $\mathrm{V}_{\mathrm{n}}$.

Proof. For each $\Phi \in \mathrm{l}^{2}(\mathbb{Z}, \mathcal{H})$, we define the function $\Psi_{\Phi}: \mathbb{Z} \rightarrow \mathcal{H}$ as

$$
\Psi_{\Phi}(\mathrm{x})=\mathrm{R}_{\mathrm{n}}(\mathrm{x}-1) \Phi(\mathrm{x}-1)+\mathrm{L}_{\mathrm{n}}(\mathrm{x}+1) \Phi(\mathrm{x}+1),
$$

by theorem3.1.4, we know that $\Psi_{\Phi} \in \mathrm{l}^{2}(\mathbb{Z}, \mathcal{H})$, and satisfies

$$
\left\|\Psi_{\Phi}\right\|_{1^{2}(\mathbb{Z}, \mathcal{H})}=\|\Phi\|_{1^{2}(\mathbb{Z}, \mathcal{H})} .
$$

Thus, we have an isometric operator $\mathrm{V}_{\mathrm{n}}: \mathrm{l}^{2}(\mathbb{Z}, \mathcal{H}) \rightarrow$ $\mathrm{l}^{2}(\mathbb{Z}, \mathcal{H})$, such that

$$
\mathrm{V}_{\mathrm{n}} \Phi=\Psi_{\Phi}, \Phi \in \mathrm{l}^{2}(\mathbb{Z}, \mathcal{H}),
$$

namely $V_{n}$ satisfies (3.6).

Let $\Phi \in \mathrm{l}^{2}(\mathbb{Z}, \mathcal{H})$, for each $\mathrm{x} \in \mathbb{Z}, \sigma \in \Gamma$, define a function $\Phi^{\sigma} \in \mathrm{l}^{2}(\mathbb{Z}, \mathcal{H})^{[10]}$ such that

$$
\Phi^{\sigma}(\mathrm{y})= \begin{cases}\mathbb{Z}_{\sigma}, & \mathrm{y}=\mathrm{x} ; \\ 0, & \mathrm{y} \neq \mathrm{x}, \mathrm{y} \in \mathbb{Z},\end{cases}
$$

which gives

$\left\langle\left[\mathrm{V}_{\mathrm{n}}^{*} \Phi\right](\mathrm{x}), \mathbb{Z}_{\sigma}\right\rangle$

$=\left\langle\left[\mathrm{V}_{\mathrm{n}}^{*} \Phi\right], \Phi^{\sigma}\right\rangle_{1^{2}(\mathbb{Z}, \mathcal{H})}$

$\left.=\left\langle\Phi, \mathrm{V}_{\mathrm{n}} \Phi^{\sigma}\right\rangle_{1^{2}(\mathbb{Z}, \mathcal{H}}\right)$

$=\sum_{\mathrm{y}=-\infty}^{\infty}\left\langle\Phi(\mathrm{y}), \mathrm{R}_{\mathrm{n}}(\mathrm{y}-1) \Phi^{\sigma}(\mathrm{y}-1)+\mathrm{L}_{\mathrm{n}}(\mathrm{y}+1) \Phi^{\sigma}(\mathrm{y}+1)\right\rangle$

$=\left\langle\Phi(\mathrm{x}+1), \mathrm{R}_{\mathrm{n}}(\mathrm{x}) \mathbb{Z}_{\sigma}\right\rangle+\left\langle\Phi(\mathrm{x}-1), \mathrm{L}_{\mathrm{n}}(\mathrm{x}) \mathbb{Z}_{\sigma}\right\rangle$

$=\left\langle\mathrm{R}_{\mathrm{n}}^{*}(\mathrm{x}) \Phi(\mathrm{x}+1)+\mathrm{L}_{\mathrm{n}}^{*}(\mathrm{x}) \Phi(\mathrm{x}-1), \mathbb{Z}_{\sigma}\right\rangle$.

Thus, we have (3.7)

Let $\Phi \in \mathrm{l}^{2}(\mathbb{Z}, \mathcal{H})$ and $\Psi=\mathrm{V}_{\mathrm{n}}^{*} \Phi$, then, by using (3.5), (3.6), for each $x \in \mathbb{Z}$, we obtain that 


$$
\begin{aligned}
& \mathrm{V}_{\mathrm{n}} \Psi(\mathrm{x}) \\
& =\mathrm{R}_{\mathrm{n}}(\mathrm{x}-1) \Psi(\mathrm{x}-1)+\mathrm{L}_{\mathrm{n}}(\mathrm{x}+1) \Psi(\mathrm{x}+1) \\
& =\mathrm{R}_{\mathrm{n}}(\mathrm{x}-1) \mathrm{V}_{\mathrm{n}}^{*} \Phi(\mathrm{x}-1)+\mathrm{L}_{\mathrm{n}}(\mathrm{x}+1) \mathrm{V}_{\mathrm{n}}^{*} \Phi(\mathrm{x}+1) \\
& =\left[\mathrm{R}_{\mathrm{n}}(\mathrm{x}-1) \mathrm{R}_{\mathrm{n}}^{*}(\mathrm{x}-1)+\mathrm{L}_{\mathrm{n}}(\mathrm{x}+1) \mathrm{L}_{\mathrm{n}}^{*}(\mathrm{x}+1)\right] \Phi(\mathrm{x}) \\
& =\Phi(\mathrm{x}) .
\end{aligned}
$$

Thus $\mathrm{V}_{\mathrm{n}} \Psi(\mathrm{x})=\Phi$, which together with the arbitrariness $\Phi \in \mathrm{l}^{2}(\mathbb{Z}, \mathcal{H})$, implies that $\mathrm{V}_{\mathrm{n}} \mathrm{V}_{\mathrm{n}}^{*}=\mathrm{I}$.

Remark 2: $V_{n}^{*} \neq V_{n}$, thus $V_{n}$ is not self-adjoint operator.

\section{Theorem 3.1.7 The Spatiotemporal}

non-homogeneous quantum random walk has a unitary representation, more precisely

$$
\Phi_{\mathrm{n}}=\left(\prod_{\mathrm{k}=0}^{\mathrm{n}-1} \mathrm{~V}_{\mathrm{k}}\right) \Phi_{0},
$$

where $\mathrm{V}_{\mathrm{k}}: l^{2}(\mathbb{Z}, \mathcal{H}) \rightarrow \mathrm{l}^{2}(\mathbb{Z}, \mathcal{H})$ is the unitary operator defined by Theorem 3.1.6.

Proof. According to (3.6), we have

$$
\Phi_{\mathrm{n}}(\mathrm{x})=\left[\mathrm{V}_{\mathrm{n}-1} \Phi_{\mathrm{n}-1}\right](\mathrm{x}), \mathrm{x} \in \mathrm{Z}, \mathrm{n} \geq 1,
$$

which implies (3.8).

\subsection{Characterization in Tensor Space}

As is seen, The Spatiotemporal non homogeneous quantum random walk is formulated in the function space $\mathrm{l}^{2}(\mathbb{Z}, \mathcal{H})$. In the present subsection, we reformulate it in the sensor space $\mathrm{l}^{2}(\mathbb{Z}) \otimes \mathcal{H}$, which is isomorphism to $l^{2}(\mathbb{Z}, \mathcal{H})$ in the sensor of unitary isomorphism.

Definition 3.2.1 Let $\mathrm{f} \in \mathrm{l}^{2}(\mathbb{Z}), \mathrm{h} \in \mathcal{H}$, we define a function $\Phi_{\mathrm{f}, \mathrm{h}}: \mathbb{Z} \rightarrow \mathcal{H}$, such that

$$
\Phi_{\mathrm{f}, \mathrm{h}}(\mathrm{x})=\mathrm{f}(\mathrm{x}) \mathrm{h}, \mathrm{x} \in \mathbb{Z},
$$

which means that $\left\{\Phi_{\mathrm{f}, \mathrm{h}} \mid \mathrm{f} \in \mathrm{l}^{2}(\mathbb{Z}), \mathrm{h} \in \mathcal{H}\right\}$ is a complete set in space $\mathrm{l}^{2}(\mathbb{Z}, \mathcal{H})$.

Lemma 3.2.2 Let $\mathrm{J}: \mathrm{l}^{2}(\mathbb{Z}, \mathcal{H}) \rightarrow \mathrm{l}^{2}(\mathbb{Z}) \otimes \mathcal{H}$, be the canonical unitary isomorphism. Then, J satisfies that

$$
\mathrm{J} \Phi_{\mathrm{f}, \mathrm{h}}=\mathrm{f} \otimes \mathrm{h}, \quad \mathrm{f} \in \mathrm{l}^{2}(\mathbb{Z}), \mathrm{h} \in \mathcal{H} .
$$

Lemma 3.2.3 Define a operator $S: l^{2}(\mathbb{Z}) \rightarrow l^{2}(\mathbb{Z})$, satisfies that

$$
\operatorname{Sf}(x)=f(x-1), x \in Z, f \in l^{2}(\mathbb{Z}) .
$$

namely $\mathrm{S}$ is a unitary operator, then we have

$$
\begin{array}{r}
\mathrm{S}=\sum_{\mathrm{x} \in \mathbb{Z}}\left|\delta_{\mathrm{x}}><\delta_{\mathrm{x}-1}\right|, \\
\mathrm{S}^{-1}=\sum_{\mathrm{x} \in \mathbb{Z}}\left|\delta_{\mathrm{x}}><\delta_{\mathrm{x}+1}\right|,
\end{array}
$$

where $\left\{\delta_{\mathrm{x}} \mid \mathrm{x} \in \mathbb{Z}\right\}$ is the canonical OBN of $\mathrm{l}^{2}(\mathbb{Z})$ and $\left|\delta_{\mathrm{x}}><\delta_{\mathrm{x}-1}\right|$ the Dirac operator.

According to the above definition and lemma, we can give the representation in tensor space $l^{2}(\mathbb{Z}) \otimes \mathcal{H}$ of unitary operators $\mathrm{V}_{\mathrm{n}}$ in space $\mathrm{l}^{2}(\mathbb{Z}, \mathcal{H})$.

For each $n \geq 0$, we denote by $G_{n}$ the counterpart of $V_{n}$ in the tensor space $l^{2}(\mathbb{Z}) \otimes \mathcal{H}$, namely

$$
\mathrm{G}_{\mathrm{n}}=\mathrm{JV}_{\mathrm{n}} \mathrm{J}^{-1} \text {. }
$$

Then $G_{n}$ is a unitary operators onl $l^{2}(\mathbb{Z}) \otimes \mathcal{H}$.

Theorem 3.2.4 Let $n \geq 0$. Then, the unitary operator $G_{n}$ has a structure of the following form

$$
\begin{aligned}
G_{\mathrm{n}}= & \sum_{\mathrm{x} \in \mathbb{Z}}\left[\left|\delta_{\mathrm{x}}><\delta_{\mathrm{x}-1}\right| \otimes \mathrm{R}_{\mathrm{n}}(\mathrm{x}-1)+\right. \\
& \left.\left|\delta_{\mathrm{x}}><\delta_{\mathrm{x}+1}\right| \otimes \mathrm{L}_{\mathrm{n}}(\mathrm{x}+1)\right],
\end{aligned}
$$

where the operator series converge in the sense of strong topology.

Proof. For each $x \in \mathbb{Z}$, combine (3.9) and (3.11), we have

$$
\begin{aligned}
& V_{n} \Phi_{f, h}(x) \\
& =R_{n}(x-1) \Phi_{f, h}(x-1)+L_{n}(x+1) \Phi_{f, h}(x+1 \\
& =[(S f)(x)] R_{n}(x-1) h+\left[\left(S^{-1} f\right)(x)\right] L_{n}(x+1) h \\
& =\left[\Phi_{S f, R_{n}(x-1) h}+\Phi_{S^{-1} f, L_{n}(x+1) h}\right](x),
\end{aligned}
$$

namely $\mathrm{V}_{\mathrm{n}} \Phi_{\mathrm{f}, \mathrm{h}}=\Phi_{\mathrm{Sf}, \mathrm{R}_{\mathrm{n}}(\mathrm{x}-1) \mathrm{h}}+\Phi_{\mathrm{S}^{-1} \mathrm{f}_{\mathrm{L}}(\mathrm{x}+1) \mathrm{h}}$.

For each $f \otimes h \in l^{2}(\mathbb{Z}) \otimes \mathcal{H}$. by definition 3.2.2 we have $\mathrm{J}^{-1}(\mathrm{f} \otimes \mathrm{h})=\Phi_{\mathrm{f}, \mathrm{h}}$, then

$$
\begin{aligned}
& V_{n} J^{-1}(f \otimes h)=J V_{n} \Phi_{f, h} \\
& =J \Phi_{S f, R_{n}(x-1) h}+J \Phi_{S^{-1} f, L_{n}(x+1) h} \\
& =\left(S \otimes R_{n}(x-1)+S^{-1} \otimes L_{n}(x+1)\right)(f \otimes h) .
\end{aligned}
$$

Namely $\mathrm{JV}_{\mathrm{n}} \mathrm{J}^{-1}=\mathrm{S} \otimes \mathrm{R}_{\mathrm{n}}(\mathrm{x}-1)+\mathrm{S}^{-1} \otimes \mathrm{L}_{\mathrm{n}}(\mathrm{x}+1)$.

Let $G_{n}=J V_{n} J^{-1}$, by using (3.11), we obtain that

$\mathrm{G}_{\mathrm{n}}$

$=\mathrm{JV}_{\mathrm{n}} \mathrm{J}^{-1}$

$=\sum_{\mathrm{x} \in \mathbb{Z}}\left[\left|\delta_{\mathrm{x}}><\delta_{\mathrm{x}-1}\right| \otimes \mathrm{R}_{\mathrm{n}}(\mathrm{x}-1)+\left|\delta_{\mathrm{x}}><\delta_{\mathrm{x}+1}\right| \otimes \mathrm{L}_{\mathrm{n}}(\mathrm{x}+1)\right]$

together with $G_{n}=J V_{n} J^{-1}$, we know $G_{n}^{*}=\left(J V_{n} J^{-1}\right)^{*}=$ $\mathrm{JV}_{\mathrm{n}}^{*} \mathrm{~J}^{-1}$,

which implies that

$$
G_{n} G_{n}^{*}=J V_{n} J^{-1} J V_{n}^{*} J^{-1}=J V_{n} V_{n}^{*} J^{-1}=I,
$$

where I denotes the identity operator on $\mathrm{l}^{2}(\mathbb{Z}, \mathcal{H})$. Similarly, we can prove the formulas $G_{n}^{*} G_{n}=I$, namely $G_{n}$ is unitary operator. 
Remark 3: $G_{n} \neq G_{n}^{*}$, thus $G_{n}$ not self-adjoint operator.

Let $\mathrm{W}_{\mathrm{n}} \in \mathrm{l}^{2}(\mathrm{Z}) \otimes H$, and $\mathrm{W}_{\mathrm{n}}=\mathrm{J} \Phi_{\mathrm{n}}$. Due to $\Phi_{\mathrm{n}}$ means the state of the walk in $l^{2}(\mathbb{Z}, \mathcal{H})$ at time $n \geq 0$, thus $W_{n}$ be the state of the walk at time $n \geq 0$, together with $G_{n}=J V_{n} J^{-1}$ we have

$$
\mathrm{W}_{\mathrm{n}+1}=\mathrm{G}_{\mathrm{n}} \mathrm{W}_{\mathrm{n}} .
$$

Theorem 3.2.5 Let the walk in tensor space $\mathrm{l}^{2}(\mathbb{Z}, \mathcal{H})$ has unitary representation as following

$$
\begin{aligned}
& \mathrm{W}_{\mathrm{n}}=\left(\prod _ { \mathrm { k } = 0 } ^ { \mathrm { n } - 1 } \sum _ { \mathrm { x } \in \mathbb { Z } } \quad \left[\quad\left|\delta_{\mathrm{x}}><\delta_{\mathrm{x}-1}\right| \otimes \mathrm{R}_{\mathrm{n}}(\mathrm{x}-\right.\right. \\
& \text { 1) } \left.\left.+\left|\delta_{\mathrm{x}}><\delta_{\mathrm{x}+1}\right| \otimes \mathrm{L}_{\mathrm{n}}(\mathrm{x}+1)\right]\right) \mathrm{W}_{0} \text {, }
\end{aligned}
$$

where $n \geq 1$ and $\mathrm{w}_{0} \in \mathrm{l}^{2}(\mathbb{Z}, \mathcal{H})$ is the initial state of the walk.

Proof. By using (3.14),(3.15),we have

$$
\mathrm{W}_{\mathrm{n}}=\left(\prod_{\mathrm{k}=0}^{\mathrm{n}-1} \mathrm{G}_{\mathrm{k}}\right) \mathrm{W}_{0}, \quad \mathrm{n} \geq 0,
$$

which implies (3.16).

\section{Limit Probability Distribution}

In this section, we force on exploring limit probability distribution of Spatiotemporal nonhomogeneous quantum random walk.

we know the evolution of the walk is governed by equation

$$
\begin{aligned}
& \Phi_{(n+1)}(\mathrm{x}) \\
& =\mathrm{R}_{\mathrm{n}}(\mathrm{x}-1) \Phi_{\mathrm{n}}(\mathrm{x}-1)+\mathrm{L}_{\mathrm{n}}(\mathrm{x}+1) \Phi_{\mathrm{n}}(\mathrm{x}+1) .
\end{aligned}
$$

Clearly, for each $\mathrm{n} \geq 0$, the function $\mathrm{x} \mapsto\|\Phi(\mathrm{x})\|^{2}$ makes a probability distribution on $\mathbb{Z}$. Thus, the probability that the quantum walker is at position $\mathrm{x} \in \mathbb{Z}$ at time $\mathrm{n} \geq 0$ starting from the initial state $\Phi_{0}$ is naturally defined to be.

$$
\mathrm{P}\left\{\mathrm{X}_{\mathrm{n}}=\mathrm{x}\right\}=\|\Phi(\mathrm{x})\|^{2} .
$$

\subsection{Probability volatility formula}

Lemma 4.1.1[12] Let $n \geq 0$ and $\sigma \subset \mathbb{N}_{n-1}$. Then, for each $\eta \in \mathrm{H}_{\mathrm{n}-1}$, one have

$$
\left\|\mathrm{L}_{\sigma} \mathrm{R}_{\mathbb{N}_{\mathrm{n}-1} \mid \sigma} \eta\right\|^{2}=\frac{1}{2^{\mathrm{n}}}\|\eta\|^{2},
$$

where $\mathbb{N}_{\mathrm{n}-1}=\{0,1,2 \cdots \mathrm{n}-1\}, \mathrm{n} \geq 0$, and for all $\mathrm{n} \geq 0$, have $\mathbb{N}_{\mathrm{n}-1} \in \Gamma$.

Theorem 4.1.2 Let $\left(\Phi_{n}\right)_{n \geq 0}$ be the state sequence of Spatiotemporal non-homogeneous quantum random walk, $\Phi_{0} \in \mathrm{l}^{2}(\mathrm{Z}, \mathrm{H})$ with $\left\|\Phi_{0}\right\|_{1^{2}}^{2}=1$. Then, for all $\mathrm{n} \geq 1$, it holds that

$$
\begin{aligned}
\Phi_{\mathrm{n}}(\mathrm{x})= & \mathrm{e}^{-\mathrm{i}(2 \#(\sigma)-\mathrm{n}) \xi(\mathrm{x}-1)} \times \\
& \sum_{\mathrm{n}-1 \notin \sigma, \sigma \subset \mathrm{N}_{\mathrm{n}-1}} \mathrm{~L}_{\sigma} \mathrm{R}_{\mathrm{N}_{\mathrm{n}-1} \backslash \sigma} \Phi_{0}(\mathrm{x}+2 \# \sigma-\mathrm{n})+ \\
& \mathrm{e}^{-\mathrm{i}(2 \#(\sigma)-\mathrm{n}) \xi(\mathrm{x}+1)} \times \\
& \sum_{\mathrm{n}-1 \in \sigma, \sigma \subset \mathrm{N}_{\mathrm{n}-1}} \mathrm{~L}_{\sigma} \mathrm{R}_{\mathrm{N}_{\mathrm{n}-1} \backslash} \backslash \Phi_{0}(\mathrm{x}+2 \# \sigma-\mathrm{n}),
\end{aligned}
$$

where $\mathrm{x} \in \mathbb{Z}$.

Proof. We use the method of mathematical induction. For $\mathrm{n}=1$, it has

$$
\begin{aligned}
& \Phi_{1}(\mathrm{x}) \\
= & \mathrm{IR}_{0}(\mathrm{x}-1) \Phi_{0}(\mathrm{x}-1)+\mathrm{IL}_{0}(\mathrm{x}+1) \Phi_{0}(\mathrm{x}+1) \\
= & \mathrm{e}^{\mathrm{i} \xi(\mathrm{x}-1)} \mathrm{L}_{\varnothing} \mathrm{R}_{0} \Phi_{0}(\mathrm{x}-1)+\mathrm{e}^{-\mathrm{i} \xi(\mathrm{x}+1)} \mathrm{R}_{\emptyset} \mathrm{L}_{0} \Phi_{0}(\mathrm{x}+1) \\
= & \mathrm{e}^{-\mathrm{i}(2 \# \sigma-1) \xi(\mathrm{x}-1)} \sum_{\substack{0 \notin \sigma, \sigma \subset \mathbb{N}_{0}}} \mathrm{~L}_{\sigma} \mathrm{R}_{\mathbb{N}_{0} \backslash \sigma} \Phi_{0}(\mathrm{x}+2 \# \sigma-1)+ \\
& \mathrm{e}^{-\mathrm{i}(2 \# \sigma-1) \xi(\mathrm{x}+1)} \sum_{\substack{0 \in \sigma_{1} \\
\sigma \subset \mathbb{N}_{0}}} \mathrm{~L}_{\sigma} \mathrm{R}_{\mathbb{N}_{0} \backslash \sigma} \Phi_{0}(\mathrm{x}+2 \# \sigma-1),
\end{aligned}
$$

which shows that formula (4.2) holds for $n=1$. Now assume that formula (4.2) holds for $\mathrm{n}=\mathrm{k}+1$, we have

$$
\begin{aligned}
& \Phi_{\mathrm{k}+1}(\mathrm{x}) \\
& =\mathrm{R}_{\mathrm{k}}(\mathrm{x}-1) \Phi_{\mathrm{k}}(\mathrm{x}-1)+\mathrm{L}_{\mathrm{k}}(\mathrm{x}+1) \Phi_{\mathrm{k}}(\mathrm{x}+1) .
\end{aligned}
$$

Note that the induction assumption implies

$$
\begin{aligned}
\mathrm{R}_{\mathrm{k}}(\mathrm{x}-1) \Phi_{\mathrm{k}}(\mathrm{x}-1) \\
=\mathrm{IR}_{\mathrm{k}}(\mathrm{x}-1) \Phi_{0}(\mathrm{x}-1) \\
=\mathrm{e}^{\mathrm{i} \xi(\mathrm{x}-1)} \mathrm{L}_{\varnothing} \mathrm{R}_{0} \Phi_{0}(\mathrm{x}-1) \\
=\mathrm{e}^{-\mathrm{i}(2 \# \sigma-(\mathrm{k}+1)) \xi(\mathrm{x}-1)} \sum_{\sigma \subset \mathbb{N}_{\mathrm{k}-1}} \mathrm{~L}_{\sigma} \mathrm{R}_{\mathbb{N}_{\mathrm{k}} \backslash \sigma} \Phi_{0}(\mathrm{x}+2 \# \sigma-1)+ \\
\quad \mathrm{e}^{-\mathrm{i}(2 \# \sigma-1) \xi(\mathrm{x}+1)} \sum_{0 \in \sigma, \sigma \subset \mathbb{N}_{0}} \mathrm{~L}_{\sigma} \mathrm{R}_{\mathbb{N}_{0} \backslash \sigma} \Phi_{0}(\mathrm{x}+2 \# \sigma-1),
\end{aligned}
$$

which shows that formula (4.2) also holds for $\mathrm{n}=\mathrm{k}+1$.

\subsection{Probability Distribution}

Theorem 4.2.1 Let the initial state $\Phi_{0} \in \mathrm{l}^{2}(\mathbb{Z}, \mathcal{H})$, as following

$$
\Phi_{0}(\mathrm{x})= \begin{cases}\mathbb{Z}_{\emptyset}, & \mathrm{x}=0 \\ 0, & \mathrm{x} \neq 0, \mathrm{x} \in \mathbb{Z},\end{cases}
$$

for all $\mathrm{n} \geq 1$, one has

$$
\begin{gathered}
P\left\{X_{n}=x\right\}=\left\|\Phi_{n}(x)\right\|^{2}= \\
\begin{cases}\frac{1}{2^{n}}\left(\begin{array}{l}
n \\
j
\end{array}\right), & x=n-2 j, 0 \leq j \leq n ; \\
0, & \text { otherwise. }\end{cases}
\end{gathered}
$$


Proof. By using (4.2), one has

$$
\begin{aligned}
& \mathrm{P}\left\{\mathrm{X}_{\mathrm{n}}=\mathrm{x}\right\} \\
& =\left\|\Phi_{\mathrm{n}}(\mathrm{x})\right\|^{2} \\
& =\left\|\mathrm{e}^{-\mathrm{i}(2 \# \sigma-\mathrm{n}) \xi(\mathrm{x}-1)}\right\|^{2} \sum_{\substack{\mathrm{n}-1 \notin \sigma, \sigma \subset \mathbb{N}_{n-1}}} \| \mathrm{L}_{\sigma} \mathrm{R}_{\mathbb{N}_{\mathrm{n}-1} \backslash \sigma} \Phi_{0}(+2 \# \sigma \\
& -\mathrm{n}) \|^{2} \\
& =\left\|\mathrm{e}^{-\mathrm{i}(2 \# \sigma-\mathrm{n}) \xi(\mathrm{x}+1)}\right\|^{2} \sum_{\substack{\mathrm{n}-1 \in \sigma, \sigma \subset \mathbb{N}_{n-1}}} \| \mathrm{L}_{\sigma} \mathrm{R}_{\mathbb{N}_{n-1} \backslash \sigma} \Phi_{0}(\mathrm{x}+2 \# \sigma \\
& -\mathrm{n}) \|^{2},
\end{aligned}
$$

together with (4.1), then has

$$
\begin{aligned}
& P\left\{X_{n}=x\right\} \\
& =\sum_{\substack{n-1 \notin \sigma, \sigma \subset \mathbb{N}_{n-1}}} \frac{1}{2^{n}}\left\|\Phi_{0}(x+2 \# \sigma-n)\right\|^{2}+ \\
& =\sum_{\substack{n-1 \in \sigma, \sigma \subset \mathbb{N}_{n-1}}} \frac{1}{2^{n}}\left\|\Phi_{0}(x+2 \# \sigma-n)\right\|^{2} \\
& =\sum_{k=0}^{n} \frac{1}{2^{n}}\left\|\Phi_{0}(x+2 \# \sigma-n)\right\|^{2} \\
& =\frac{1}{2^{n}} \sum_{k=0}^{n}\left(\begin{array}{l}
n \\
k
\end{array}\right)\left\|\Phi_{0}(x+2 k-n)\right\|^{2} \\
& =\left\{\begin{array}{l}
\frac{1}{2^{n}}\left(\begin{array}{l}
n \\
j
\end{array}\right), \quad x=n-2 j, 0 \leq j \leq n ; \\
0, \quad \text { otherwise },
\end{array}\right.
\end{aligned}
$$

namely (4.4) holds.

Lemma 4.2.2[10] Let $\Phi_{0} \in \mathrm{l}^{2}(\mathbb{Z}, \mathcal{H})$ and $\Phi_{0}=\mathrm{Z}_{\emptyset}$. Then,

$$
\frac{x_{n}}{\sqrt{n}} \Rightarrow \mathbb{N}(0,1),
$$

namely the probability distribution of $\frac{x_{n}}{\sqrt{n}}$ converges in law to the standard Gaussian distribution as $\mathrm{n} \rightarrow 0$.

Theorem 4.2.3 Let the initial state $\Phi_{0} \in \mathrm{l}^{2}(\mathbb{Z}, \mathcal{H})$ be such that

$$
\Phi_{0}(\mathrm{x})= \begin{cases}\alpha \mathrm{Z}_{\varnothing}+\beta \mathrm{Z}_{0}, & \mathrm{x}=0 \\ 0, & \mathrm{x} \neq 0, \mathrm{x} \in \mathbb{Z}\end{cases}
$$

where $\alpha, \beta \in \mathbb{C}$ 且 $|\alpha|^{2}+|\beta|^{2}=1$. Then for all $n \geq$ 1 , it holds that

$$
\begin{aligned}
& P\left\{X_{n}=x\right\} \\
& =\left\|\Phi_{n}(x)\right\|^{2} \\
& = \begin{cases}\frac{1}{2^{n}}\left[\left(\begin{array}{c}
n-1 \\
j
\end{array}\right)|\alpha-\beta|^{2}+\left(\begin{array}{c}
n-1 \\
j-1
\end{array}\right)|\alpha+\beta|^{2}\right], & x=2 j-n, 0 \leq j \\
0, & \text { otherwise. }\end{cases}
\end{aligned}
$$

where $\left(\begin{array}{c}n-1 \\ -1\end{array}\right)=\left(\begin{array}{c}n-1 \\ n\end{array}\right)=0$ and $\left(\begin{array}{l}0 \\ 0\end{array}\right)=1$.

Proof. We also use the method of mathematical induction. For $\mathrm{n}=1$, it has $\Phi_{1}(\mathrm{x})=\mathrm{R}_{0}(\mathrm{x}-1) \Phi_{0}(\mathrm{x}-$ 1) $+\mathrm{L}_{0}(\mathrm{x}+1) \Phi_{0}(\mathrm{x}+1)$.

For all $\mathrm{x} \in \mathbb{Z} \backslash\{-1,1\}$, one has $\Phi_{1}(\mathrm{x})=0$ and

$$
\begin{aligned}
& \Phi_{1}(-1)=\mathrm{L}_{0}(0) \Phi_{0}(0)=\mathrm{e}^{-\mathrm{i} \xi(0)} \frac{1}{2}(\alpha-\beta)\left(\mathrm{Z}_{0}-\mathrm{Z}_{\varnothing}\right) \\
& \Phi_{1}(1)=\mathrm{R}_{0}(0) \Phi_{0}(0)=\mathrm{e}^{\mathrm{i} \xi(0)} \frac{1}{2}(\alpha+\beta)\left(\mathrm{Z}_{0}+\mathrm{Z}_{\varnothing}\right) .
\end{aligned}
$$

namely for all $\mathrm{x} \in \mathbb{Z} \backslash\{-1,1\}$, such that $\mathrm{P}\left\{\mathrm{X}_{1}=\mathrm{x}\right\}=0$ and

$$
\begin{aligned}
\mathrm{P}\left\{\mathrm{X}_{1}=-1\right\} & \\
& =\left\|\mathrm{e}^{-\mathrm{i} \xi(0)} \frac{1}{2}(\alpha-\beta)\left(\mathrm{Z}_{0}-\mathrm{Z}_{\varnothing}\right)\right\|^{2} \\
& =\frac{1}{4}|\alpha-\beta|^{2}\left\|\left(\mathrm{Z}_{0}-\mathrm{Z}_{\emptyset}\right)\right\|^{2}=\frac{1}{2}|\alpha-\beta|^{2}, \\
\mathrm{P}\left\{\mathrm{X}_{1}=1\right\} & \\
& =\left\|\mathrm{e}^{\mathrm{i} \xi(0)} \frac{1}{2}(\alpha+\beta)\left(\mathrm{Z}_{0}+\mathrm{Z}_{\varnothing}\right)\right\|^{2} \\
& =\frac{1}{4}|\alpha+\beta|^{2}\left\|\left(\mathrm{Z}_{0}+\mathrm{Z}_{\varnothing}\right)\right\|^{2}=\frac{1}{2}|\alpha+\beta|^{2},
\end{aligned}
$$

which shows that formula (4.7) holds for $\mathrm{n}=1$. Now assume that formula (4.7) holds for $\mathrm{n}=\mathrm{k} \geq 1$. Then, for $\mathrm{n}=\mathrm{k}+1$, by using theorem 3.2 and theorem 4.3 in literature [10], then has

$$
\begin{aligned}
& \left\|\Phi_{\mathrm{k}+1}(\mathrm{x})\right\|^{2} \\
= & \left\|\mathrm{e}^{\mathrm{i} \xi(\mathrm{x}-1)} \mathrm{R}_{\mathrm{k}} \Phi_{\mathrm{k}}(\mathrm{x}-1)\right\|^{2}+ \\
& \left\|\mathrm{e}^{-\mathrm{i} \xi(\mathrm{x}+1)} \mathrm{L}_{\mathrm{k}}(\mathrm{x}+1) \Phi_{\mathrm{k}}(\mathrm{x}+1)\right\|^{2} \\
= & \frac{1}{2}\left\|\Phi_{\mathrm{k}}(\mathrm{x}-1)\right\|^{2}+\frac{1}{2}\left\|\Phi_{\mathrm{k}}(\mathrm{x}+1)\right\|^{2},
\end{aligned}
$$

namely

$$
\mathrm{P}\left\{\mathrm{X}_{\mathrm{k}+1}=\mathrm{x}\right\}=\frac{1}{2} \mathrm{P}\left\{\mathrm{X}_{\mathrm{k}}=\mathrm{x}-1\right\}+\frac{1}{2} \mathrm{P}\left\{\mathrm{X}_{\mathrm{k}}=\mathrm{x}+1\right\} .
$$

When $x=2 j-(k+1), 0 \leq j \leq k+1$, note that the induction assumption implies 


$$
\begin{aligned}
& P\left\{X_{k+1}=2 j-(k+1)\right\} \\
& =\frac{1}{2} P\left\{X_{k}=2(j-1)-k\right\}+\frac{1}{2} P\left\{X_{k}=2 j-k\right\} \\
& =\frac{1}{2^{k+1}}\left[\left(\begin{array}{c}
k \\
j-1
\end{array}\right)|\alpha+\beta|^{2}+\left(\begin{array}{c}
k \\
j
\end{array}\right)|\alpha-\beta|^{2}\right] .
\end{aligned}
$$

When $x \notin 2 j-(k+1), 0 \leq j \leq k+1$, we have

$$
\begin{aligned}
& x-1 \notin\{2 j-k \mid 0 \leq j \leq k\}, \\
& x+1 \notin\{2 j-k \mid 0 \leq j \leq k\},
\end{aligned}
$$

namely

$$
\begin{aligned}
& P\left\{X_{k+1}=x\right\} \\
& =\frac{1}{2} P\left\{X_{k}=x-1\right\}+\frac{1}{2} P\left\{X_{k}=x+1\right\} \\
& =0
\end{aligned}
$$

which shows that formula (4.7) also holds for $\mathrm{x}=\mathrm{k}+$ 1 .

Corollary 4.2.4 Let the initial state of the walk $\Phi_{0} \in \mathrm{l}^{2}(\mathrm{Z}, \mathrm{H})$ as the theorem4.2.3 shows, the random variable has probability distribution as following

$$
\frac{x_{n}}{\sqrt{n}} \Rightarrow \mathbb{N}(0,1) .
$$

\section{Conclusions}

The coin operators of Spatiotemporal nonhomogeneous quantum random walk are $\mathrm{L}_{\mathrm{n}}(\mathrm{x})=\mathrm{e}^{-\mathrm{i} \xi(\mathrm{x})} \frac{1}{2}\left(\partial_{\mathrm{n}}^{*}+\partial_{\mathrm{n}}-\mathrm{I}\right) \quad$ and $\quad \mathrm{R}_{\mathrm{n}}(\mathrm{x})=$ $\mathrm{e}^{\mathrm{i} \xi(\mathrm{x})} \frac{1}{2}\left(\partial_{\mathrm{n}}^{*}+\partial_{\mathrm{n}}+\mathrm{I}\right)$, which based on the cion operators of $\mathrm{W}-\mathrm{Y}$ quantum random walk $\mathrm{L}_{\mathrm{n}}=\frac{1}{2}\left(\partial_{\mathrm{n}}^{*}+\partial_{\mathrm{n}}-\mathrm{I}\right)$ and $\mathrm{R}_{\mathrm{n}}=\frac{1}{2}\left(\partial_{\mathrm{n}}^{*}+\partial_{\mathrm{n}}+\mathrm{I}\right)$, adding a random function $\mathrm{e}^{\mathrm{i} \xi(\mathrm{x})}$, makes the whole model have spatial inhomogeneity, which causes a certain random interference to the evolution of the model. But by prove the probability distribution under a kind of initial state, it is prove that the space-time inhomogeneous quantum random walk has the same limit as the classical random walk, which means although the random environment causes a certain random interference to the space-time inhomogeneous quantum random walk model, it has no effect on its spatial probability distribution.

\section{REFERENCE}

[1] Aharonov Y, Davidovich L, Zagury N. Quantum random walks[J]. Physical Review A, 1993,48: 1687-1690.

[2] Konno N. A new type of limit theorems for the one-dimensional quantum random walk[J]. Journal of the Mathematical Society of Japan, 2005,57(4): 1179- 1195
[3] Kempe J. Quantum random walks: an introductory overview[J]. Contemporary Physics, 2003,44(4): 307 - 327.

[4] Venegas-Andraca S.E. Quantum walks: a comprehensive review[J]. Quantum Information Processing, 2012, 11(5): 1015-1106.

[5] Konno N. Quantum walks and quantum cellular autumata[M]. Germary: Lecture Notes in Computer Science, 2008,5191:12 - 21.

[6] Mastue K, Ogurisu O, Segawa E. Quantum walks on simplicial complexes[J]. Quantum Information Processing, 2016, 15(5): $1865-1896$

[7] Suzuki A. Asymptotic velocity of a position-dependent quantum walk[J]. Quantum Information Processing, 2016,15: 103 - 119.

[8] Segawa E, Suzuki A. Generator of an abstract quantum walk[J]. Quantum Studies:Mathematics and Foundations, 2016,3: 11-30.

[9] Wang C.S, Chai H.F, Lu Y.C. Discrete-time quantum Bernoulli noise[J]. Journal of Mathematical Physics, 2010,51: 053528.

[10] Wang C.S, Ye X.J. Quantum walk in terms of quantum Bernoulli noise[J]. Quantum Information Processing, 2016, 15: 1879-1908.

[11] Wang C, Wang C.S. Higher-Dimensional Quantum Walk in Terms of Quantum Bernoulli Noises[J]. Entropy, 2020,22(5): 504.

[12] Wang C.S, Wang C, Ren S.L, et al. Open quantum random walk in terms of quantum Bernoulli noise[J]. Quantum Information Processing, 2018,17(3): 46

[13] Wada K. A weak limit theorem for a class of long-range-type quantum walks in $1 \mathrm{~d}[\mathrm{~J}]$. Quantum Information Processing, 2020,19(2)

[14] Privault N. Stochastic analysis of Bernoulli pricesses[J]. Probability Surveys, 2008,5 (1): 435-483.

Meng Li, School of Mathematics and Statistics, Northwest Normal University, Lanzhou, China, Mobile, 86-18298486202.

Caishi Wang, School of Mathematics and Statistics, Northwest Normal University, Lanzhou, China.

Rui Fu, School of Mathematics and Statistics, Northwest Normal University, Lanzhou, China. 